\title{
WELLNESS IN YOUR POCKET: UPDATE YOUR HEALTH
}

\author{
Susana Orrego, Karine Posada, Ana M. Ortiz and María M. Yepes \\ Seguros SURA Colombia \\ Carrera 64 B \# 49 A 30 Medellín, Colombia
}

\begin{abstract}
To build a system of sustainable healthcare, a private health insurance company in Latin America developed custom programs focused on achieving better solutions and delivering enhanced healthcare value to their members. The program developed is called "Update your health" (in Spanish "Tu Bienestar al Día") which means a way to keep members demographic and health status updated. The program, which use Information and Communication Technologies, enable members to manage their health risks by monitoring their health status and mitigating any risk. This is achieved through coordinated activities and help improve their health and quality of life. The insurance company take advantage of each contact with members to solve their health concerns and integrate their health information with their risk management. This paper describes the "Update your health" program five months after its implementation among members who required preventive services through screening tests, imaging, and medical consultation.
\end{abstract}

\section{KEYWORDS}

Patient Empowerment, e-Health, Risk Management, Healthcare Improvement

\section{INTRODUCTION}

"Update your Health" is a program that use the Information and Communication Technologies (ICTs) to promote healthy habits such as receiving necessary screening, and enable patients to know their health status and manage or mitigate their disease risks. The program allows the members to order screening tests, make appointments and other services through virtual access. The program uses members age, gender and comorbidities to define their needs for preventive or treatment services. This objective of this paper was to examine the effectiveness of the "Update your health" program five months after its implementation in a private insurance company in Colombia, South America.

\section{BODY OF PAPER}

This was a descriptive study which used members who seek screening tests, imaging, and medical consultation in a private insurer in Colombia between November 30, 2018 and March 12, 2019.

Members $(n=211,327)$ who were deemed to need at least one the health service (lipid profile, glycemic blood levels, creatinine, prostate antigen, papanicolaou, mammography, total abdominal ultrasound, assessment by general practitioner, gynecologist or urologist) based on their risk profile as assessed using age, gender and comorbidities; were contacted using the programs "Marketing Cloud" platform to participate in the program. There were informed that preventive and medical services could be provided to them without the need to attend a face-to-face consultation. Once members agreed to participate in the program, health personnel contacted them through phone calls to schedule appointments to undertake the required service needed. Once the services were performed, the virtual health management team contacted those members and provided them with medical advice on test results, additional tests needed, the need to schedule additional appointments, and other recommendations as appropriate. 
A total of 4,456 members agreed to participate in the program, of whom 843 were contacted by phone. Out of the 843 members contacted by phone, 107 completed the required medical services, 31 had completed some tests but were waiting to receive others, 3,981 were waiting to receive the services they required, and 337 decline to receive any service offered. Of the services provided, 1,342 members results came back normal, 418 had one or more abnormal results for lipid profile, glycemic blood levels, creatinine and prostate antigen.

\section{CONCLUSION}

The implementation of "Update your Health" program has had an impact on the health management of members through easy access to care, reduce wait times to receiving care, increased satisfaction, mobility, and savings in out-of-pocket expenses. "Update your Health" program is an innovative tool which focused on the insured and their life cycle, seeking to impact early detection of diseases in order to keep members healthier over time. Moreover, it has a direct impact on access to care, providing ICTs tools for health management virtually without the need for a face-to-face consultation.

\section{ACKNOWLEDGEMENT}

The program has achieved its three aims: improve health outcomes of members, reduce cost for the insurer and members, and increase members satisfaction. The program could be implemented in other countries. However, the challenge will be with being able to secure members information and providing the right information at the right time for each member.

\section{REFERENCES}

Hamine, S. et al, 2015. Impact of mHealth chronic disease management on treatment adherence and patient outcomes: a systematic review. Journal of medical Internet research, 17(2), e52. doi:10.2196/jmir.3951.

Yara M. Asi. Et al, 2018. The role of digital health in making progress toward Sustainable Development Goal (SDG) 3 in conflict-affected populations. International Journal of Medical Informatics, Vol. 114, pages 114-120.

Meskó, B. et al, 2017. Digital health is a cultural transformation of traditional healthcare. mHealth, 3, 38. doi:10.21037/mhealth.2017.08.07.

Sanjeev, B. et al, 2016. Mobile technology and the digitalization of healthcare. European Heart Journal, Vol. 37, issue 18 , pages $1428-1438$.

McConnell, M. et al, 2018 Mobile Health Advances in Physical Activity, Fitness, and Atrial Fibrillation: Moving Hearts. Journal of the American College of Cardiology, Vol. 71, Issue 23, pages 2691 - 270. 